\title{
ESTUDIANTES PARA MAESTROS: REFLEXIONES SOBRE LA INSTRUCCIÓN EN LOS NÚMEROS RACIONALES POSITIVOS
}

\author{
José María Gairín Sallán \\ Universidad de Zaragoza
}

\begin{abstract}
RESUMEN: Los estudiantes para Maestros tienen importantes dificultades de comprensión de los números racionales. Buena parte de estas dificultades están producidas por las decisiones didácticas que se tomaron en el proceso instructivo de estos estudiantes, proceso en el que se priorizó el significado de la fracción como relación entre la parte y el todo.

Caracterizar este tipo de dificultades, así como las peculiaridades del conocimiento de los Maestros en formación, constituye la primera parte de este trabajo.

En la segunda parte se ofrecen alternativas didácticas que ayuden a los futuros Maestros a superar las dificultades mencionadas: el trabajo con el modelo medida les permite reelaborar sus conocimientos sobre el sistema de los números racionales positivos, mientras que el trabajo con el modelo cociente les permite fortalecer las conexiones entre las notaciones fraccionaria y decimal.
\end{abstract}

ABSTRACT: Future Primary Education teachers find it extremely difficult to understand rational numbers. The difficulties they have to face are mainly due to a previous learning process, a process which used to consider better to learn the meaning of fractions as being related to the part and the whole.

The first part of this paper is focused on the study of such difficulties and also on the special characteristics of the knowledge adquired by these future teachers.

In the second part alternative methods are proposed in order to help these future teachers to overcome the above mentioned difficulties: Working with a measure model allows them to re-elaborate their knowledge of the positive rational number system, whereas working with a quotient model allows them to reinforce the connections between fraction and decimal notations.

PALABRAS CLAVE: Aprendizaje, matemáticas, enseñanza superior, estudiante universitario.

KEYWORDS: Learning, mathematics, higher education, university student. 


\section{INTRODUCCIÓN}

Como formadores de Maestros estamos interesados, con carácter general, en estudiar las dificultades de comprensión que tienen estos estudiantes sobre los distintos significados que componen el concepto de número racional y también sobre su estructura como sistema o conjunto de entes, relaciones y operaciones (Hiebert, 1993).

Igualmente estamos interesados en estudiar alternativas didácticas que permitan poner de manifiesto las dificultades de comprensión detectadas en los futuros Maestros, con la finalidad de evitar el riesgo de retroalimentar los errores de los escolares como consecuencia de las dificultades de comprensión de sus profesores. Una vez superado este primer nivel se podrá continuar con la formación didáctica de los estudiantes para Maestro haciendo que reflexionen sobre el proceso de adquisición de los conocimientos matemáticos (Brown, 1993; Sowder, Philipp, Flores y Schappelle, 1995).

El punto de partida de este artículo lo constituyen una serie de fenómenos advertidos en las producciones de los Maestros en formación; estos fenómenos son consecuencia de un proceso instructivo en el que se prima el significado de la fracción como relación parte-todo, y en el que las expresiones decimales se presentan como entes abstractos, como números no medida. Se completa así una primera parte del trabajo en la que se dan respuestas a cuestiones relacionadas con el proceso de enseñanza-aprendizaje de los números racionales: ¿cuáles son las características de los conocimientos matemáticos que utilizan? y iqué dificultades de comprensión se detectan en las producciones de los Maestros en formación?

En la segunda parte del trabajo, y con la intención de mejorar la formación de los futuros Maestros, ofrecemos una propuesta educativa para incrementar la comprensión de los números racionales que tienen estos estudiantes; los puntos básicos de esta propuesta son la concreción de un modelo en el que significar tanto a las fracciones como a las expresiones decimales, conceptualizar a las fracciones y a las expresiones decimales como expresiones de cantidades de magnitud, posibilitar que los estudiantes reelaboren su mapa conceptual sobre los números racionales positivos y establecer vías de conexión entre las notaciones fraccionaria y decimal.

\section{Conocimientos previos de los Maestros en formación}

Desde nuestra preocupación por ayudar a los estudiantes a superar sus dificultades de comprensión de los números racionales nos propusimos analizar las concepciones que tienen los futuros Maestros en aspectos muy concretos: significados de las fracciones y de las expresiones decimales, significado de las relaciones de orden y de la densidad respecto del orden, y conexiones entre las representaciones fraccionaria y decimal.

Con esta finalidad realizamos, en el curso 1997-98, una encuesta entre los estudiantes de $2^{\text {o }}$ curso de la Diplomatura de Maestro (Especialidad de Educación Primaria) de la Universidad de Zaragoza. La encuesta se realizó en la primera sesión del curso, antes de comenzar el desarrollo de la asignatura. Las respuestas, por tanto, indican las concepciones previas de los alumnos sobre los números racionales. Se encuestó a 47 estudiantes, de cuyas respuestas sobre los númeroa racionales señala- 
mos las características más sobresalientes, acompañadas de los datos que han permitido tal caracterización (Gairín, 1999):

Característica 1. Los estudiantes para Maestro tienen un significado casi exclusivo de la fracción como relación parte-todo; además, este significado se asocia a un modelo físico.

Pregunta: Escribe todo lo que entiendes cuando ves el símbolo 5/7

Respuestas: como primera respuesta el 59\% de los estudiantes hacen una lectura de los símbolos (fracción, número racional, o cinco séptimos); mientras que el $32 \%$ de ellos responde como relación parte-todo (parte tomada de una tarta, de una barra de helado, ..); y el $9 \%$ de las respuestas son erróneas. Como segunda respuesta aparece la relación parte-todo (45\%), el 9\% mencionan la fracción como cociente (dividir el numerador entre denominador), y el resto contienen nombres o errores. Ninguna de las respuestas menciona los significados de medida, operador o razón.

Característica 2. La notación decimal se reconoce exclusivamente con significado numérico, no se asocia a cantidades de magnitud ni se sustenta en el uso de modelos.

Pregunta: Escribe todo lo que entiendes cuando ves el símbolo 2'04

Respuestas: el 53\% de las respuestas contiene exclusivamente los términos número decimal o número racional; otro $32 \%$ recurren al sistema de numeración ( 2 unidades y 4 centésimas); el $13 \%$ contiene respuestas descriptivas de amplio espectro (número positivo, número compuesto de unidades y decenas...); el resto de las respuestas son erróneas. En ninguna de las respuestas dadas se hace referencias a modelos físicos similares a los utilizados en las fracciones.

Característica 3. La relación entre las notaciones fraccionaria y decimal se establece de forma exclusiva mediante procesos algorítmicos.

Pregunta: Escribe distintas formas de justificar que los números 0,375 y $3 / 8$ son iguales

Respuestas: el 74\% utiliza como justificación el algoritmo de la división del numerador entre el denominador, mientras que un $11 \%$ lo justifica mediante la escritura como fracción del decimal; el resto de las respuestas son erróneas o evasivas (con una regla de tres o con expresiones algebraicas):

$$
0,375 \cdot x=\frac{3}{8} \cdot x, \operatorname{luego} \frac{0,375 \cdot 8}{3}=\frac{x}{x} ; \frac{0,375 \cdot 8}{3}=1
$$

Característica 4. Las relaciones de orden entre fracciones no se justifican en modelos, simplemente se utilizan técnicas de cálculo.

Pregunta: Escribe distintas formas de justificar que la fracción 3/5 es mayor que $4 / 7$ 
Respuestas: el orden entre fracciones se establece a través de su representación decimal (47\%) o por la búsqueda de fracciones equivalentes con igual denominador (34\%). Es significativo el $17 \%$ de respuestas erróneas que aparecen: Partimos de la base que si tenemos una unidad y si la dividimos en 5 partes iguales, éstas serán mayores que si las dividimos en 7.

Característica 5. Solamente la tercera parte de los estudiantes establece relaciones de orden entre números decimales aplicando el principio del valor posicional.

Pregunta: Escribe distintas formas de justificar que el número 0,468 es mayor que 0,35

Respuestas: el 36\%, utiliza la técnica de "observar el primer decimal"; en el $13 \%$ de las respuestas los decimales se transforman en naturales (multiplicando por 1000 los dos decimales); el 13\% de las respuestas convierten los decimales en fracciones y comparan las fracciones; en el 18\% de las respuestas se utilizan argumentos numéricos (restar o dividir los números a comparar, buscar el más próximo a 0 o a 1). Resulta llamativo que el $9 \%$ de los alumnos no de respuesta alguna y que para el $11 \%$ de los encuestados no haya que justificar la "evidencia".

Característica 6. Los estudiantes para Maestro no admiten la densidad respecto del orden de los números racionales; la topología del conjunto de los números racionales es la misma que la de los números naturales

Pregunta: Escribe los números siguientes a los que se dan:
a) 2,3 ;
b) 0,567 ;
c) $1,222 \ldots$
d) $\frac{1}{2}$
e) -2
f) $0,9999 \ldots$

Respuesta: en todos los casos los estudiantes han señalado el número siguiente al propuesto con argumentos como los siguientes:

- Con números decimales la respuesta mayoritaria es la de considerar como número natural a la parte decimal: el siguiente de 2,3 es 2,4 (66\% de las respuestas), el siguiente de 0,567 es 0,568 (85\% de las respuestas).

- Con números periódicos las respuestas surgen de considerar como un número natural a una parte del periodo: el siguiente a 1,222... es 1,23 (34\% de las respuestas), 1,3 (26\% de las respuestas), o 1,223 (19\% de las respuestas).

- En el caso de las fracciones la tendencia mayoritaria es la de actuar sobre el denominador: el siguiente a $1 / 2$ es 1 (entendiendo que $1=1 / 1 ; 36 \%$ de las respuestas); también son destacables las respuestas obtenidas desde la conversión de las fracciones en decimales: la fracción siguiente a $1 / 2$ es $3 / 5$ (26\% de las respuestas; entendiendo que el proceso ha sido $1 / 2=0,5$; el siguiente de 0,5 es 0,6, por tanto, la fracción siguiente a $1 / 2$ es $3 / 5=0,6)$.

- En el caso de números enteros casi la totalidad de los estudiantes no contemplan la posibilidad de interpretarlos como números racionales; pero considerados como números enteros hay un porcentaje significativo de respuestas $(21 \%)$ que mantienen el orden de los naturales: el siguiente de -2 es -3 
Estos resultados vienen a confirmar los de otros estudios acerca de los conocimientos de los estudiantes para Maestros sobre los números racionales (Llinares, Sánchez y García, 1994; Llinares y Sánchez, 1996; Philippou y Christou, 1994; Pinto y Tall, 1996; Post, Harel, Behr y Lesh, 1991; Simón, 1993), así como la constatación de que tales resultados vienen determinados por la instrucción recibida por los alumnos (Gairín, 1999):

- los estudiantes priorizan, de forma casi exclusiva, el significado de la fracción como relación parte-todo;

- las expresiones decimales no se significan en el contexto de las magnitudes medibles;

- las fracciones y las expresiones decimales se conciben como tópicos desconectados;

- las relaciones de orden solamente se justifican desde las manipulaciones de números abstractos;

- la traslación de significados desde los números naturales a los números racionales obstaculiza la conceptualización de la densidad respecto del orden de los números racionales.

\section{Las producciones de los estudiantes para Maestros}

Señaladas las características del conocimiento de los futuros Maestros sobre el número racional positivo nuestra hipótesis es que buena parte de estas concepciones y errores tiene su origen en obstáculos didácticos, en las dificultades y errores que se originan como consecuencia del modo en que se presentan los conceptos matemáticos (Brousseau, 1983). Más concretamente sostenemos que el origen de muchas de las dificultades de comprensión de estos estudiantes hay que situarlo en un proceso educativo que priorizó el conocimiento de la fracción sustentado en el significado parte-todo (Gairín, 1999, pág. 6).

Nos proponemos hacer una reflexión sobre ocho fenómenos relacionados con la comprensión de los Números Racionales y que hemos detectado en nuestra actividad docente con Maestros en formación. Resulta pertinente hacer esta reflexión porque ello nos va a permitir establecer alternativas didácticas que ayuden a los estudiantes a superar sus dificultades de comprensión. Y esta tarea es prioritaria en la formación de educadores matemáticos puesto que en las actuaciones de los futuros profesores se manifiestan sus creencias y concepciones sobre la naturaleza de la matemática, sobre su enseñanza y sobre su aprendizaje, aspectos que condicionan la enseñanza que impartirán y, consecuentemente, lo que aprenderán sus alumnos (Ball, 1993; Thompson y Thompson, 1996; Llinares y Sánchez, 1996)

Nuestro interés, por tanto, se centra en destacar aquellos fenómenos de comprensión que reflejan las limitaciones, dificultades y errores que aparecen cuando estos estudiantes resuelven tareas relacionadas con el currículum de Educación Primaria; estos fenómenos se describen tal y como los presentan los estudiantes para Maestro (Gairín, 1999). 
Fenómeno 1: El significado de la fracción es un conocimiento inestable.

Ejemplo a: Se propone a los estudiantes la cuestión siguiente:

¿Qué fracción representa la parte sombreada de la figura?
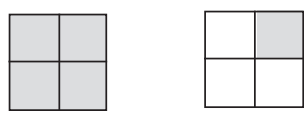

Figura 1

La respuesta de los alumnos no es unánime: para unos es $5 / 4$ y para otros es $5 / 8$. Es más, ninguno de los alumnos que da una de las respuestas es capaz de rebatir la otra respuesta.

Ejemplo b: Se propone a los estudiantes justificar cuál de las fracciones $3 / 4$ y $6 / 5$ es la mayor

Estudiante A: Represento las dos fracciones sobre una misma unidad, porque de lo contrario no puedo compararlas (muestra el siguiente dibujo):
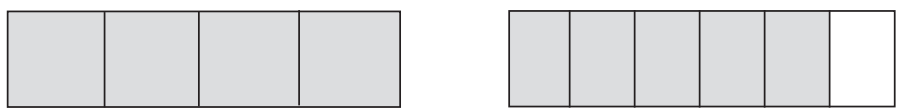

Figura 2

Vemos que, ante la necesidad de controlar un aspecto de la tarea, el alumno ha convertido la fracción 6/5 en la fracción 5/6, lo que implica un cambio sustancial en el significado de la fracción. Ejemplos como estos sustentan nuestra idea de que el significado de la fracción como relación parte-todo es menos estable de lo que se presuponía, pues puede modificarse sustancialmente en función de las exigencias de la tarea. Este fenómeno ha de evitarse ampliando el significado parte-todo con otros significados del número racional

Fenómeno 2: Las ideas están fuertemente influenciadas por la percepción visual.

Ejemplo: comparar las fracciones $3 / 5$ y $4 / 7$, justificando los resultados obtenidos.

Estudiante B: después de haber representado las dos fracciones, no puedo estar seguro de cuál de las partes rayadas es la más grande (aporta este dibujo):
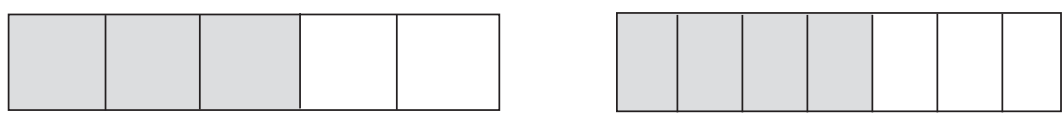

Figura 3 
Este estudiante representa las dos fracciones a comparar sobre "todos" iguales, pero la comparación no puede justificarse más que con la percepción visual de las áreas destacadas, que solo es posible si son manifiestamente distinguibles. Vemos, por tanto, que el razonamiento de éste alumno queda limitado a la percepción visual, y que tal forma de actuación es útil en muchos casos, pero resulta inoperante al trabajar con fracciones muy próximas. En la formación de Maestros este fenómeno hay que evitarlo pues los educadores matemáticos han de ser tecnólogos, han de conocer las técnicas y también las razones de su existencia; de lo contrario en la formación de los escolares se priorizará el uso de las técnicas.

Fenómeno 3: Se tiende a sustituir los conceptos por alguna de las técnicas asociadas.

Ejemplo: ¿Son equivalentes las fracciones $5 / 12$ y 10/24?, ¿por qué?.

Estudiante C: si que son equivalentes porque al multiplicar (o dividir) el numerador y el denominador de una fracción por un mismo número se obtiene la otra fracción equivalente.

Ahora bien, ¿qué ocurre si se les plantea la siguiente situación?

Pedro retira 5 bombones de una caja de 12, retira 5/12 de la caja.

Ana retira 10 bombones de una caja de 24, retira 10/24 de la caja.

¿Son equivalentes $5 / 12$ y $10 / 24$ ?

Pues ocurre que se encuentra en un dilema que no sabe resolver: de una parte, acaba de reconocer que las fracciones $5 / 12$ y $10 / 24$ son equivalentes pero, de otra parte, observa que Pedro retira 5 bombones mientras que Ana retira 10 bombones. Este ejemplo nos permiten afirmar que éste alumno entiende que conocer el concepto de equivalencia de fracciones significa conocer la técnica de comprobación de dicho concepto y no las formas distintas de expresar la misma cantidad de magnitud. Entendemos que el conocimiento matemático abarca tanto el dominio de los conceptos como el de las técnicas asociadas, y que ésta idea deben mantenerla en sus tareas profesionales; de lo contrario, sus alumnos recibirán una instrucción parcial.

Fenómeno 4: Los conocimientos de los estudiantes sobre los números racionales suelen estar limitados al uso de reglas.

Ejemplo: Justifica el algoritmo de la suma con las fracciones $2 / 3$ y $4 / 5$.

Estudiante D: Es necesario representar las fracciones sobre las misma unidad.
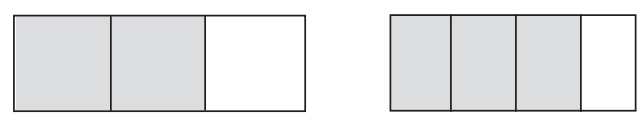

Figura 4 
Veo que representan el mismo trozo pero luego a la hora de sumarlo no sabría cómo hacerlo; porque aquí una parte equivale a 4 trocitos de un tamaño y aquí esta otra equivale a 2 trocitos de otro tamaño... Pero es que no se, no lo se hacer.

Este tipo de respuestas ponen de manifiesto que éste estudiantes utiliza procedimientos de cálculo que no están conceptualmente justificados, simplemente son hechos aprendidos; el conocimiento sobre los algoritmos no incluye su fundamentación, simplemente le preocupa su funcionamiento y el buen uso que se haga del mismo. El avance y extensión de las calculadoras lleva a enfatizar la instrucción tanto en los conceptos como en las técnicas. Consecuentemente la formación de los futuros Maestros demanda conocer los distintos conceptos sobre los números racionales, así como conocer las razones matemáticas que soportan las distintas técnicas.

\section{Fenómeno 5: La descontextualización de la medida obstaculiza la resolución de problemas.}

Ejemplo: De una cesta de manzanas se retiran todas menos siete; quedan 43 manzanas. ¿Cuántas había?

Estudiante E: El problema está mal planteado puesto que 1/2 - 7 es negativo.

Entendemos que este tipo de respuestas procede de una concepción de las fracciones como números no medida, como entes abstractos alejados del mundo de las magnitudes; en consecuencia la esencia de la información se limita al valor numérico y no a la cantidad de medida a que se refiere. Los problemas constituyen un amplio campo de hechos físicos que se modelizan con los números racionales. Estos hechos están indudablemente unidos al campo de las magnitudes medibles y, en consecuencia, el controlar la magnitud con la que se trabaja y la unidad de medida utilizada resultan esenciales para alcanzar la modelización correcta.

Fenómeno 6: El orden entre expresiones decimales está fuertemente influenciado por el orden entre números naturales.

Ejemplo: Encuentra todos las expresiones decimales situadas entre $4{ }^{\prime} \overline{27}$ y $4^{\prime} \overline{28}$ justificando la respuesta

Estudiante $\mathrm{F}$ : entre $4^{\prime} \overline{27}$ y $4^{\prime} \overline{28}$ no hay ningún número porque en el primer caso es $27,27,27 \ldots$ y en el segundo $28,28,28 \ldots$ y no se puede poner ningún número en medio porque detrás del 27 va el 28

La no modelización de las expresiones decimales, el que el estudiante no disponga de un soporte físico en el que sustentar sus argumentaciones sobre objetos del mundo real, limita la conceptualización de las expresiones decimales periódicas como pares de números enteros separados por una coma; y desde esta situación se manipulan los entes numéricos de acuerdo con reglas conocidas y sin considerar la distinta naturaleza de los números. En la actuación de este estudiante se observa que la estructura topológica de los números naturales se traslada a otro conjunto numérico, lo que constituye un obstáculo para alcanzar una correcta comprensión de la densidad respecto del orden de los números racionales. 
Ciertamente los alumnos no suelen cometer errores si trabajan con expresiones decimales con un número finito de cifras. Ahora bien, en la preparación de los futuros Maestros es importante proponer tareas en las que haya que trabajar el orden con expresiones periódicas, pues ello favorecerá sus conocimientos sobre la naturaleza del orden en los números racionales, así como sobre las peculiaridades de la densidad respecto al orden.

Fenómeno 7: En el cálculo con números periódicos se crean algoritmos a partir de los que conocen de los números naturales y de los números decimales.

Ejemplo: Señalamos algunas técnicas utilizadas por estudiantes al operar con números periódicos:

- Sumar como números decimales y poner de periodo tantas cifras como las del sumando con más cifras en el periodo: $0^{\prime} 3 \overline{814}+9^{\prime} 6 \overline{29}=10^{\prime} 0 \overline{104}$

- Multiplicar como números decimales y poner como cifras del periodo la suma de las cifras de los periodos: $4^{\prime} \overline{2} \times 0^{\prime} \overline{543}=2^{\prime} \overline{2806}$

- Utilizar el algoritmo de la división extendiendo el número de cifras del periodo hasta que aparece un 0 en el resto; el cociente es un número decimal

$$
3^{\prime} 0686868 \ldots: 3=1^{\prime} 0228956
$$

Puesto que los estudiantes consideran a las expresiones decimales como entes abstractos, suelen readaptar los algoritmos de cálculo de números decimales para operar los números periódicos; pero esta readaptación no pueden justificarla ni desde la caracterización de los operandos como cantidades de magnitud, ni desde el conocimiento de los algoritmos que utilizan. En consecuencia, los estudiantes utilizan algoritmos inadecuados de cálculo con números periódicos, pero tienen limitaciones para evaluar la correcta aplicación de los mismos o para detectar el origen de sus errores. Es importante, para la formación de Maestros, que conozcan situaciones para las que no existen (o son muy complejos) algoritmos de cálculo; de este modo les podremos ayudar a contextualizar las operaciones en el mundo de las magnitudes, así como a controlar el sentido que tienen las técnicas de cálculo y la viabilidad de las mismas en distintas situaciones.

Fenómeno 8: Los números periódicos dificultan la formulación de situaciones problemáticas coherentes.

Ejemplo a: Enuncia un problema que se resuelva con la operación $0^{\prime} 3 \overline{814}+9^{\prime} 6 \overline{29}$

Estudiante G: Un corredor lleva una velocidad constante de $0^{\prime} 38 \overline{14}$ y un compañero una velocidad constante de $9^{\prime} 6 \overline{29}$; se quieren sumar las velocidades para luego obtener la media.

Ejemplo b: Enuncia un problema que se resuelva con la operación $6^{\prime} 1 \overline{37}: 6$

Estudiante $\mathrm{H}$ : Un corredor lleva una velocidad constante de $6^{\prime} 1 \overline{37}$ para recorrer 6 kilómetros, ¿cuál es la velocidad en cada uno de los kilómetros?

Una dilatada experiencia como aprendices de matemáticas parece perfilar una dinámica de resolución de problemas en la que el énfasis se sitúa en la realización 
correcta de los cálculos, sin preocuparse de las peculiaridades de las magnitudes a las que se refieren, ni al contexto en el que sitúan dichas magnitudes. Poner a los estudiantes para Maestro ante la formulación de problemas es una actividad interesante por cuanto les exige pensar en los números periódicos como cantidades de magnitud, también les exige controlar las peculiaridades de las magnitudes con las que trabajan, así como establecer relaciones coherentes entre cantidades de distintas magnitudes.

\section{PROPUESTAS PARA LA FORMACIÓN INICIAL DE MAESTROS}

Caracterizados los conocimientos previos sobre los Números Racionales y analizadas sus consecuencias en las producciones de futuros Maestros, queremos ofrecer vías para diseñar tareas instructivas que mejoren la formación inicial de estos profesionales docentes: utilizar un mismo modelo para conceptualizar a las expresiones fraccionaria y decimal; significar a la fracción como cociente partitivo; fortalecer las conexiones entre las notaciones fraccionaria y decimal; y posibilitar que los estudiantes reelaboren su mapa conceptual sobre los números racionales. Sobre estos aspectos, y sobre la metodología a utilizar nos extendemos en los siguientes apartados.

\section{Caracterizar un modelo para el aprendizaje de los números racionales positivos}

Para que el proceso de construcción del conocimiento en el ámbito escolar sea efectivo es preciso disponer de un medio físico y natural que sirva para la formación de conceptos y que sirva también como área de aplicación de los mismos; así, a partir de la imagen y combinando pensamiento con experiencia, la formación de ideas sobre los números racionales positivos aparece conectada.

En el caso de los estudiantes para Maestro cabe pensar que estos deberían tener desarrollada una gran capacidad de abstracción, que no necesitan de modelos físicos sobre los que construir ideas matemáticas; sin embargo, nuestra experiencia como docentes indica que esta situación no es general, que hay un importante número de estudiantes con serias dificultades para razonar sobre ideas abstractas; es más, en el caso de las fracciones el significado queda fuertemente vinculado al modelo en que se concibió, de tal manera que la mayoría de estos estudiantes siguen mencionando las tartas o las barras de helado para expresar sus ideas sobre fracciones.

Utilizamos el término modelo para designar un entorno físico con variables bien definidas, estable frente a interacciones con el mundo exterior, y que permite las acciones de los sujetos (Gairín, 2001). En esta noción de modelo tienen cabida ideas similares que reciben distintas denominaciones: así ocurre con los Modelos Concretos de Gagatsis y Patronis (1990), que se definen como objetos tridimensionales con los que se representan ideas matemáticas; o en el caso de las Experiencias Básicas de Lesh, Post y Behr (1987) que se interpretan como manipulaciones de hechos reales y sucesos que sirven de contexto general para resolver situaciones problemáticas.

- Desde la posición del aprendiz, el empleo de modelos tienen gran utilidad en la construcción del conocimiento matemático, por cuanto que:

- permiten la aprehensión sensorial de hechos y relaciones matemáticas mediante la manipulación de objetos físicos o la simulación de acciones; 
- facilitan la construcción o interpretación de los sistemas de representación que comunican los resultados producidos al actuar sobre los objetos;

- facilitan la comprensión de las relaciones sintácticas y semánticas de los sistemas de representación empleados;

- sirven como apoyo y contraste de la certeza o falsedad de las relaciones simbólicas que se establecen a través de los sistemas de representación;

- facilitan la resolución de situaciones problemáticas cuando éstas se formulan en términos de los objetos del modelo.

- Desde la posición del profesor el modelo constituye la herramienta que se ofrece al alumno con una clara intencionalidad educativa: proporcionarle un entorno físico sobre el que pueda actuar y reflexionar para que, mediante esta interacción, avance en la construcción del conocimiento cuyo aprendizaje se promueve. Por tanto, la propia intencionalidad del recurso didáctico obliga al profesor a diseñar y establecer el modelo que permita hacer explícitos aquellos aspectos que considera relevantes del concepto matemático que se quieren enseñar; en consecuencia, el modelo que propone el profesor viene condicionado por sus conocimientos sobre el concepto matemático objeto de la instrucción. En suma, corresponde al profesor tomar, cuando menos, dos cautelas en la elección del modelo: de una parte, analizar los aspectos del concepto que explicita el modelo y los que oculta u obstaculiza; y, de otra parte, cómo transmitir al alumno una caracterización precisa del modelo que destaque los aspectos relevantes de los objetos, la acciones que se pueden realizar y las características del resultado que se han de considerar.

Nos pronunciamos por un proceso instructivo en el que el modelo juega un papel esencial tanto para el que enseña como para el que aprende y, en consecuencia, la caracterización del modelo constituye un aspecto básico en el diseño de la instrucción. En el caso de los números racionales los modelos para el aprendizaje vienen definidos por cuatro variables (Gairín, 2001): una magnitud medible, unos objetos en los que se puedan medir cantidades de las magnitudes consideradas, unas acciones sobre los objetos que provocan modificaciones en la cantidad de magnitud y una técnica para realizar las acciones.

Una vez definido el modelo, hay que comunicar los resultados de las acciones realizadas en el mismo. Surge así la noción de sistema de representación como el modo de expresar y simbolizar determinadas estructuras numéricas mediante unos signos, unas reglas y unos enunciados (Castro, Rico, Romero, 1997); la notación fraccionaria y la notación decimal son los dos sistemas de representación más usuales en el caso de los números racionales. Entendemos que el uso y gestión de sistemas de representación desempeña un papel central en la comprensión de las ideas matemáticas, puesto que un análisis profundo de las características sintácticas y semánticas que subyacen en el sistema de representación utilizado fortalece la comprensión de las mencionadas ideas matemáticas.

Queda así caracterizado un proceso instructivo en el que el modelo constituye el medio para la construcción de ideas matemáticas, en el que la comunicación de esas ideas implica la construcción de sistemas de representación estrechamente relacio- 
nados con el modelo (al menos en su fase inicial), y en el que la evaluación semántica de expresiones formuladas con los sistemas de representación se facilita a través del modelo (Lesh, 1997; Gairín, 1999).

\section{Significar la fracción como medida}

Una parte importante de las dificultades y errores de los estudiantes hay que situarla en un conocimiento casi exclusivo de la fracción como relación parte-todo, significado que se introduce como recurso didáctico (Escolano y Gairín, 2004): pensamos que el origen del significado parte-todo habría que situarlo en la práctica educativa, habría que ubicarlo entre los recursos didácticos creados por necesidades del proceso de la enseñanza y del aprendizaje de las matemática.

Además, puesto que la génesis histórica del número racional hay que situarla en el contexto de la medida y de la razón de medidas, abandonamos la idea de partetodo y situamos la instrucción sobre el número racional en la medida de cantidades de magnitud que no contienen un número entero de veces la unidad de medida. De éste modo, el número racional surge del fraccionamiento de la unidad de medida, del uso de una nueva unidad de medida que es un submúltiplo de la unidad inicial; el número racional expresa la cantidad de veces que se ha utilizado una, o varias, subunidades cuyos tamaños también aparecen de forma explícita.

Por otra parte, al situarnos en el contexto de la medida potenciamos la ruptura con la idea de número, limitada al número natural, que tienen los estudiantes para Maestro, puesto que se encuentran con situaciones en las que los naturales se muestran ineficaces, situaciones que sugieran la necesidad de construir un nuevo sistema de números. Esta idea de número racional asociada a la medida está presente en las más antiguas culturas y ha formado parte de la instrucción sobre éste tipo de números:

Cuando se trata de apreciar cantidades menores que aquella que se toma por unidad, nos vemos en la necesidad de dividir dicha unidad en cierto número de partes iguales, y referir la cantidad que se va a medir a una de estas partes, dando origen de este modo a las fracciones o quebrados ordinarios, a las fracciones decimales o a los números complejos... Vemos, pues, que las fracciones o quebrados tienen su origen o resultan de la medición de una cantidad con una unidad mayor que aquella; lo cual se consigue dividiendo la unidad en un cierto número de partes iguales, y viendo las veces que una de estas partes está contenida en la cantidad que se quiere medir.

(Sánchez Vidal, B., 1866, pp. 122-123)

\section{Reelaborar el mapa conceptual de los estudiantes sobre los números racionales}

A la instrucción sobre el conjunto de los números racionales se le concede gran importancia en los currícula españoles de matemáticas y buena parte de la responsabilidad del proceso de enseñanza-aprendizaje recae en los Maestros, para cuyo desarrollo profesional resulta fundamental el fortalecer sus conocimientos sobre este conjunto numérico.

La consecución de este objetivo exige que los estudiantes para Maestro reelaboren su mapa conceptual sobre los números racionales, que reflexionen sobre la naturaleza y funciones de estos números, que comprendan las diferencias y similitudes 
entre estos números y los números naturales, y que reflexionen sobre el significado y sobre el papel que juegan cada tipo de número. Deben entender que la unidad constituye el elemento básico del recuento en los números naturales, mientras que en los números racionales la unidad cumple dos funciones muy diferenciadas: por una parte, es la unidad divisible que constituye el elemento esencial para la comparación de números; $y$, por otra parte, considerado como elemento unidad u operador unidad, constituye la base conceptual para la formación del inverso de la multiplicación (Kieren, 1993).

Desde estas consideraciones, nuestra propuesta didáctica presenta las siguientes peculiaridades:

1. Puesto que existe una relación entre el modelo de aprendizaje utilizado y la noción de fracción que se promueve en quien lo utiliza, proponemos el modelo definido por las siguientes variables (Gairín, 2001):

- Las magnitudes son longitud y superficie.

- Los objetos son tiras de papel (longitud) y polígonos de cartón (superficie).

- La acción que se propone es la de medir.

- La técnica de medida consiste en una sola partición de la unidad, en la creación de una única subunidad de medida. El tamaño de esta subunidad y el número entero de subunidades que contiene la cantidad a medir son los elementos necesarios para comunicar el resultado de la medida.

2. La fracción $\mathbf{m} / \mathbf{n}$ se presenta como el resultado de una medida, como la expresión del número $\mathbf{m}$ de subunidades -de tamaño $\mathbf{1 / n}$ de unidad-, que corresponde a la cantidad de magnitud medida.

De este modo, la fracción queda asociada a la medida de cantidades de magnitud realizadas con una técnica determinada, lo que nos va a permitir:

- Presentar una ruptura entre los números naturales y los racionales a partir de sus diferentes usos. Contar y medir son acciones de diferente naturaleza que demandan técnicas diferenciadas y, en consecuencia, sistemas de representación distintos.

- Potenciar un aprendizaje activo, puesto que es el propio estudiante quien tiene que decidir la elección de la subunidad de medida necesaria para efectuar la medida.

- Introducir la idea del fraccionamiento de la unidad en un número de partes iguales, idea que se hace necesaria por cuanto la unidad de medida no cabe un número entero de veces en la cantidad a medir.

- Introducir, de forma natural, las fracciones con numeradores mayores, menores o iguales que el denominador, sin más que proponer la medida de cantidades mayores, menores o iguales a la unidad.

- Posibilitar la introducción de las notaciones fraccionaria y decimal de los números racionales positivos mediante la utilización de dos técnicas de medida distintas: medir con una sola subunidad, medir en una sola fase; o medir con subunidades de la unidad o de partes de la unidad, medir en varias fases. 
- Justificar las relaciones de equivalencia y de orden entre fracciones mediante la comparación de cantidades de magnitud.

- Dar significado a las operaciones entre fracciones desde el trabajo con cantidades de magnitud y justificar los correspondientes algoritmos de cálculo.

3. La equivalencia de fracciones es un aspecto esencial para establecer relaciones y operaciones entre fracciones, así como para construir el cuerpo de los número racionales (Llinares y Sánchez, 1988, pp. 117):

La importancia de la equivalencia de fracciones se debe al papel clave que juega en diversos aspectos: en la relación de orden, en el desarrollo de los algoritmos de la suma y resta de fracciones de denominador diferentes. En un nivel más elevado, la conceptualización del número racional como clases de equivalencia de fracciones...

Atendiendo a esta importancia, en el proceso instructivo ponemos especial cuidado en que los alumnos expresen la idea de equivalencia de fracciones, y ponemos de manifiesto que tal noción no debe sustituirse por alguna de las técnicas de verificación o construcción de fracciones equivalentes. Esas técnicas aparecen en el momento en que los estudiantes tienen que verificar la igualdad de dos cantidades de magnitud expresadas de formas distintas, será la necesidad de utilizar una subunidad común la que conducirá a la justificación del correspondiente algoritmo.

4. Las relaciones de orden entre fracciones necesitan de la noción de equivalencia, pues ello permite comparar cantidades de magnitud medidas con la misma subunidad de medida. Este recurso de creación de subunidades permite avanzar la idea de densidad respecto del orden; en efecto, la búsqueda de fracciones intercaladas entre otras dos dadas permite que los alumnos conciban ideas numéricas distintas a las de los números naturales: no tiene sentido mencionar términos como siguiente o anterior a una fracción, entre dos fracciones existen otras infinitas, los números fraccionarios no permiten el recuento, etc.

5. Los conceptos de suma y resta de fracciones positivas están asociados a la agregación o disgregación de cantidades de una misma magnitud. Los correspondientes algoritmos de cálculo se justifican por la necesidad de medir ambas cantidades con la misma subunidad y, por tanto, por la necesidad de operar con fracciones equivalentes.

6. El sentido de la multiplicación de fracciones se puede, y se debe, abordar desde una doble perspectiva: la de operador y la de producto de cantidades de magnitud.

- Como operador se originan dos situaciones bien diferenciadas: si el operador es un número natural la multiplicación tiene el significado de suma reiterada de cantidades de magnitud; y si el operador es una fracción el 
sentido es el de transformación de una cantidad de magnitud, el de parte de parte de una cantidad.

- Como producto de dos cantidades de magnitud hay que analizar previamente las peculiaridades de las magnitudes intervinientes y las de la magnitud resultante. De este modo, encontramos, de una parte, que el resultado expresa alguna de las magnitudes que caracterizan los datos y, de otra parte, encontramos situaciones en las que el resultado se refiere a una magnitud distinta de las contenidas en los datos.

El algoritmo de la multiplicación de fracciones se puede construir a partir de los dos significados. En nuestro caso optamos por construir dicho algoritmo a partir de la noción de producto de longitudes, y se construye como expresión de la medida de la superficie rectangular que viene determinada por lados cuyas longitudes sean igual a los factores. De este modo, el denominador del resultado, producto de los denominadores, expresa el tamaño de la subunidad de medida que se necesita para medir la superficie; mientras que el numerador del resultado indica el número de veces que está incluida dicha subunidad en la superficie a medir. En esta construcción resultan de gran ayuda las representaciones gráficas.

7. El sentido de la división de fracciones se construye desde las nociones de multiplicación: como operador inverso o como la cantidad de magnitud que multiplicada por otra cantidad conocida nos produce una nueva cantidad de magnitud conocida.

El correspondiente algoritmo se construye desde la determinación de la longitud de una de las dimensiones de un rectángulo del que se conoce la superficie y la otra dimensión. En esta construcción resultan de vital importancia las representaciones gráficas, tal y como puede verse en Gairín (2000).

\section{Dar significado a la fracción como cociente partitivo}

Para incrementar la comprensión de los números racionales los Maestros en formación deben fortalecer sus conocimientos sobre los diferentes significados de la fracción y conectar estos con la concepción dominante de relación parte-todo (Novillis y Larson, 1980). De no ser así, se producirán importantes deficiencias en su formación: Si los estudiantes aprenden solamente la interpretación de la fracción como relación parte-todo, tienen serias limitaciones para una sólida comprensión de las fracciones (Kerslake,1986).

En nuestra propuesta contemplamos que la fracción también se signifique como cociente partitivo, entendido como la medida de la cantidad de magnitud resultante de la disgregación de una cantidad inicial de magnitud en varias cantidades iguales; significado que ya se detecta en las fracciones egipcias surgidas en el contexto de la resolución de problemas de reparto. Por tanto, entendemos la fracción como resultado de una acción realizada por el sujeto y no con un sentido de relaciones operatorias numéricas, como cociente indicado; significado que es frecuente en manuales escolares actuales y no tan actuales: la fracción se entiende como la división indicada del numerador por el denominador, cuyo cociente es el quebrado (Sánchez Vidal, B., 1866, pág. 124-125). 
Para dar significado a la fracción como cociente partitivo sugerimos un modelo definido por los siguientes valores de las variables (Gairín, 1999):

- Los objetos que se consideran son tortillas españolas de igual superficie, de forma circular e igual radio; son objetos habituales en la vida de los estudiantes y sobre los que realizan fraccionamientos en sectores circulares.

- La magnitud elegida es la superficie porque la igualdad de cantidades de superficie se controla por la igualdad de los ángulos de los sectores; igualdad que puede comprobarse por percepción visual o con el transportador de ángulos.

- La acción que se realiza sobre los objetos es la de hacer repartos igualitarios, la de disgregar unidades de magnitud en un número entero de partes de igual tamaño.

- La técnica para efectuar la acción es la de hacerlo en una sola fase: las unidades se fraccionan en tantas partes iguales como participantes, a cada uno de los cuales se les entrega una parte de cada una de las unidades a repartir.

Para cuantificar el resultado del reparto, para expresar la cantidad de magnitud de una cualquiera de las particiones efectuadas hay que utilizar el sistema de representación fraccionario $\mathbf{m} / \mathbf{n}$, que indica la cantidad (formada por $\mathrm{m}$ subunidades de tamaño $1 / \mathbf{n}$ ) de magnitud que recibe cada uno de $\mathbf{n}$ los participantes entre los que se reparten $\mathbf{m}$ unidades de magnitud.

- Desde la fracción con significado de cociente partitivo los futuros Maestros pueden reelaborar el sentido de las relaciones y operaciones entre fracciones desde una nueva concepción. Así, por ejemplo, las fracciones a/b y c/d son equivalentes si representan el mismo resultado del reparto, si los participantes en dos repartos reciben la misma cantidad de magnitud, lo que exige que $c=n a y d=n b$

- Razonar sobre la existencia de infinitas fracciones comprendidas entre otras dos posibilita el sentido de la densidad respecto del orden, que es una idea esencial para la comprensión de la estructura topológica del conjunto de los números racionales (Giménez, 1991; Post et al, 1991). Desde el significado de fracción como cociente, este razonamiento puede hacerse por vías diferentes:

- Justificar que si $\frac{a}{b}>\frac{c}{d}$, se cumple que $\frac{a}{b}>\frac{\frac{a}{b} \frac{c}{d}}{2}>\frac{c}{d}$

Basta observar que los términos extremos indican los resultados de dos repartos diferentes, las cantidades distintas que corresponden a dos individuos que han participado en repartos distintos; mientras que el término central expresa que esos individuos se juntan y se vuelven a repartir igualitariamente las cantidades que les correspondían anteriormente: para que ambos reciban la misma cantidad, esta cantidad debe ser menor que a/b y debe ser mayor que c/d. 
A partir de la desigualdad anterior, dadas dos fracciones desiguales el alumno siempre podrá encontrar otras infinitas fracciones intermedias.

- Justificar que si $\frac{a}{b}>\frac{c}{d}$, se cumple que $\frac{a}{b}>\frac{a+c}{b+d}>\frac{c}{d}$

Hay que tener en cuenta que $\mathrm{a} / \mathrm{b}$ es el resultado de repartir a unidades entre b participantes; que la cantidad recibida en ese reparto, a/b, es mayor que la recibida en el reparto de c unidades entre d individuos; y que al hacer un sólo reparto, $(a+c)$ unidades entre $(b+d)$ individuos, los participantes reciben menor cantidad que en el reparto $a / b$, pero mayor que en el reparto $\mathrm{c} / \mathrm{d}$.

A partir de la desigualdad anterior, dadas dos fracciones desiguales siempre se pueden encontrar otras infinitas fracciones intermedias.

- Si $\frac{a}{b}>\frac{c}{d}$, se construyen fracciones equivalentes $\frac{a d}{b d}>\frac{b c}{b d}$ que repre-

sentan resultados de repartos con igual número de participantes; por tanto, todas las fracciones de la forma

$$
\frac{n}{b d} \text {, siendo } a d>n>b c \text {, cumplen que } \frac{a d}{b d}>\frac{n}{b d}>\frac{b c}{b d}
$$

\section{Conceptualizar las expresiones decimales como cantidades de magnitud}

Existe un reconocimiento generalizado a la labor de Simón Stevin, como introductor de la notación decimal en Europa, aun cuando, como señala Boyer (1986, pág 402), es claro que no fue ni el inventor ni el primero que utilizó las fracciones decimales pues estas tenían una larga historia en Mesopotamia, en la antigua China, en la Arabia medieval y en la Europa renacentista. El trabajo de Stevin enfatiza las ventajas de un recurso técnico que facilita la escritura y los cálculos con fracciones, aunque debajo de la notación decimal se esconden conceptos relacionados con el sistema de numeración decimal y con el significado de las fracciones que han tardado siglos en ser entendidos y utilizados por la humanidad.

La construcción formal del conjunto de los números decimales positivos se puede hacer como extensión de los números naturales añadiendo un elemento d, tal que $10 \mathrm{~d}=1$, obteniendo un conjunto engendrado por todas las potencias de $d$, por sus productos y por sus sumas. También se puede construir el conjunto de los números decimales positivos como extensión de los números enteros buscando las soluciones de la ecuación $10^{\mathrm{n}} . \mathrm{x}=\mathrm{a}$, siendo a un número entero y $\mathrm{n}$ un número natural. (Brousseau, 1993). Sin embargo, la tendencia de los manuales escolares es la de construir las fracciones decimales positivas como una restricción de los números racionales, considerando solamente aquellos números racionales que pueden expresarse mediante una fracción decimal, y una vez construido el conjunto de las fracciones decimales se introduce el convenio que permite pasar de las fracciones decimales a la notación decimal.

Ahora bien, bajo esta técnica se oculta una relación entre fracciones ordinarias y fracciones decimales que, históricamente, no fue sencilla de establecer y que tuvo 
dificultades de aceptación: Desde luego se comprende que empleando la ley decimal en las divisiones y subdivisiones de la unidad, no siempre se podrá expresar exactamente el valor de las cantidades que apreciadas por quebrados ordinarios podría hacerse con exactitud... (Sánchez Vidal, B., 1866, pág. 158-159).

Los currícula oficiales actuales obligan a la instrucción sobre la escritura decimal de las fracciones. Esta tarea presenta dificultades importantes, dificultades que pueden justificar una práctica educativa que soslaye la incidencia en los aspectos conceptuales y que potencie los aspectos normativos sobre su utilización. Desde este supuesto resulta plausible que los alumnos generalicen el significado de las representaciones simbólicas para números naturales a fracciones, y viceversa (Marck, 1995); que el orden de los números naturales interfiera el orden de las fracciones decimales, (Resnick, et al, 1989); o que los estudiantes tiendan a aplicar reglas de los números naturales para comparar las fracciones decimales (Owens y Super, 1993).

En los manuales escolares se presentan las expresiones decimales a partir de fracciones significadas como relación parte-todo; en estas condiciones la mayor parte del conocimiento se percibe por medio de representaciones gráficas: en las representaciones gráficas el alumno debe reconocer las unidades fraccionarias decimales, establecer relaciones de las unidades fraccionarias y la unidad, y conocer y aplicar las normas de escritura de la notación decimal. Además, el alumno debe modificar las técnicas de fraccionamiento utilizadas en el trabajo con fracciones ordinarias pues ahora se exigen dos modificaciones: de una parte, los fraccionamientos no sólo se realizan sobre la unidad, también hay que hacerlos sobre partes de la unidad; de otra parte, los fraccionamientos no se hacen en el número de partes que decida el estudiante, ahora solamente se admiten fraccionamientos en 10 partes iguales.

Nuestra propuesta (Gairín, 2001b) es la de presentar las expresiones decimales como un sistema de representación surgido desde el mismo modelo en que se significaron las fracciones como cocientes partitivos. En este sentido se propone una secuencia instructiva que contemple los siguientes aspectos:

a) Utilizar como técnica el reparto por fases, con fraccionamientos siempre en 10 partes iguales y otorgando en cada fase la mayor cantidad posible de magnitud; vamos a ilustrar esta técnica con el reparto de 3 tortillas entre 4 personas Fase 1: dar a cada individuo unidades enteras, tortillas completas: es imposible porque hay más individuos que tortillas. En la primera fase cada individuo recibe 0 unidades.

Fase 2: hay que fraccionar cada tortilla en 10 partes iguales; aparecen 30 partes de tamaño 1/10 de tortilla; el número máximo de estas partes que puede recibir cada uno de los 4 participantes es 7 . En esta fase cada participante recibe 7 partes de tamaño 1/10 de tortilla y quedan 2 partes, de tamaño 1/10 de tortilla, por repartir.

Fase 3: un nuevo fraccionamiento en 10 partes iguales produce 20 partes de tamaño 1/100 de tortilla; el número máximo de partes que puede recibir cada individuo es 5; se entregan a cada participante 5 partes de tamaño 1/100 de tortilla y no queda ninguna parte por repartir. El reparto ha concluido.

El resultado del reparto, la cantidad de tortilla que recibe cada uno de los participantes, es 0 tortillas completas, 7 partes de tamaño 1/10 de tortilla y 5 partes de tamaño 1/100 de tortilla. 
b) Poner de manifiesto que el resultado del reparto viene caracterizado por la suma de partes de tamaños que son potencias de 1/10 de unidad. En el ejemplo anterior podríamos indicar el resultado como $0+7(1 / 10)+5(1 / 100)$ tortillas.

c) Facilitar la escritura del resultado teniendo en cuenta las características de nuestro sistema de numeración: atendiendo al valor posicional y a la suma de cantidades representadas por cada una de las cifras de las expresiones decimales, el resultado anterior $0+7(1 / 10)+5(1 / 100)$ se puede simbolizar como 0,75 .

d) Incidir en el significado de las expresiones decimales positivas como representaciones de cantidades de magnitud resultantes de la suma de los valores que representan cada una de las cifras de dichas expresiones.

e) Conectar la práctica del fraccionamiento antes descrito con el algoritmo de la división de números enteros, incidiendo en el significado del di videndo como número de unidades a repartir, del divisor como número de participantes y del cociente como resultado del reparto. Reflexionar sobre el paralelismo de las acciones realizadas en el reparto y de las técnicas implicadas en el algoritmo de la división.

\section{Fortalecer las conexiones entre las notaciones fraccionaria y decimal}

Las notaciones fraccionaria y decimal son sistemas simbólicos paralelos que representan los mismos conceptos; aunque para el alumno es una idea difícil de asimilar el que cualquier concepto, especialmente un número, pueda tener más de un símbolo (Owens y Super, 1993). Por tanto, para incrementar la comprensión de los números racionales hay que fortalecer conceptualmente las conexiones entre las notaciones fraccionaria y decimal: "Enseñar fracciones y decimales como tópicos separados sin proporcionar a los estudiantes oportunidades para establecer conexiones, empequeñece su desarrollo para la plena comprensión de los números racionales" (Sowder, 1995).

La secuencia instructiva habitual en el sistema educativo español contempla que los números decimales se estudien a partir de las fracciones, reproduciendo el proceso histórico que se produjo en Europa. Puesto que la fracción admite distintos significados conviene analizar las conexiones que desde cada uno de ellos se pueden establecer con la notación decimal:

- Desde el significado de relación parte-todo son débiles las conexiones entre las notaciones fraccionaria y decimal por cuanto el estudiante asocia la fracción a una parte de la unidad compuesta por partes alícuotas de la misma, mientras que las expresiones decimales exigen interpretar la fracción como agregación de partes de unidad de diferentes tamaños.

- Desde el significado de la fracción como resultado de la medida de magnitudes se puede abordar la conexión entre las notaciones fraccionaria y decimal, pero se encuentran dificultades técnicas inherentes a la continuidad de la medida, salvo que la instrucción se cimentase en la participación pasiva de los estudiantes (limitar su trabajo a la observación de representaciones gráficas), supuesto que se aleja de nuestras intenciones educativas. Este significado de la fracción es más adecuado para conectar la aritmética con la geometría y el espacio. 
- Desde el significado de razón encontramos dificultades para utilizar un mismo modelo en el que asentar los significado de fracción y de expresión decimal, puesto que hay que justificar la transformación de dos cantidades de magnitud en una sola magnitud de naturaleza diferente a las anteriores; además, esta transformación implica un fraccionamiento que tampoco se justifica con facilidad, sobre todo si está implicada la medida de contar. Sí que es un significado adecuado para conectar la lógica del razonamiento proporcional con la lógica de las magnitudes intensivas.

- Desechamos utilizar el significado de la fracción como operador porque si se utiliza un modelo con objetos discretos no se puede efectuar el fraccionamiento de los mismos; porque si el modelo utilizan magnitudes continuas hay dificultades con la continuidad de la medida; y porque si se utilizan números sin medida no se produce una conexión entre los significados de fracción y de expresión decimal. Los operadores sí que facilitan la instrucción sobre el significado del inverso de la multiplicación y sobre la composición de aplicaciones.

Se ha postulado porque la secuencia instructiva contemple significar a las fracciones como cocientes partitivos en un modelo determinado desde el que se pueden, como se ha señalado, establecer conexiones entre las notaciones fraccionaria y decimal de los números racionales positivos. Defendemos la pertinencia de la propuesta formulada por las razones siguientes:

- La acción de repartir por fases permite concebir la fracción como agregación de cantidades de magnitud de diferentes tamaños; se ofrece una nueva perspectiva de las fracciones que, desde la interpretación como relación partetodo, solamente se contemplan como adición de partes alícuotas del "todo".

- El modelo físico en el que se ha significado la fracción como cociente partitivo posibilita la significación de las expresiones decimales como otr a forma de representar cantidades de magnitud resultantes de un mismo reparto.

- La construcción del conocimiento sobre las fracciones y sobre las expresiones decimales se hace desde las reflexiones mentales efectuadas sobre las representaciones gráficas.

- La instrucción exige la participación activa de quien aprende puesto que la acción de repartir exige de una permanente toma de decisiones por parte del aprendiz: decidir sobre la necesidad de fraccionar, decidir las cantidades que corresponden a cada participante, decidir si hay que finalizar el reparto, etc.

\section{Utilizar una metodología adecuada}

Nuestro propósito es el de ofrecer a los futuros profesores oportunidades para explorar, conjeturar, comprobar y reflexionar sobre el sentido de los números racionales contemplados desde un significado diferente: las notaciones fraccionaria y decimal expresan cantidades de magnitud resultantes de repartos igualitarios. Se ofrece una nueva perspectiva de los números racionales desde la que ayudaremos a superar la actual situación en la que muchos profesores en ejercicio tienen dificultades con cuestiones conceptuales y procedimentales de los números racionales y no son capaces de resolver problemas más que de modo procedimental (Post, Harel, Behr y Lesh, 1991). 
Atendiendo a las recientes investigaciones sobre psicología del aprendizaje, nos proponemos situar al estudiante para Maestro como agente principal en la construcción de sus conocimientos, que en las sesiones de clase se prioricen los trabajos individuales: "Las presentaciones claras por si mismas son inadecuadas para sustituir los errores por ideas claras. Lo que los estudiantes construyen por si mismos, aunque pueda ser inadecuado, está con frecuencia lo suficientemente arraigado como para no ser erradicado con una explicación seguida de unos pocos ejercicios" (National Research Council, 1989, pág. 60).

Teniendo en cuenta que los estudiantes para Maestro tienen conocimientos previos sobre los números racionales, debemos dar oportunidades para que estos estudiantes exploren y razonen desde sus conocimientos personales tanto sobre los conceptos como sobre los procedimientos; para ello, hay que hacer propuestas que les fuercen a poner en evidencia los errores adquiridos en experiencias previas y a construir nuevos conocimientos sobre las relaciones y conexiones que subyacen en este conjunto numérico; posteriormente deben reflexionar sobre el nuevo conocimiento y la forma en que lo han adquirido (Sowder, Bezuk y Sowder, 1993).

Nuestra propuesta es utilizar una metodología en la que prime el trabajo individual de los estudiantes, puesto que una presentación formal de la materia específica y el trabajo formal con números no anima a los estudiantes a preocuparse de la reconstrucción de su "concepto imagen" desde la definición de número racional (Pinto y Tall, 1996, pág. 151). Estos trabajos individuales deben contener situaciones problemáticas que tengan sentido para ellos y que sean generadoras de conflictos que favorezcan el sentido del número y no habilidades rutinarias y reglas para su aplicación: "es a través de la resolución de problemas como un concepto cualquiera adquiere sentido para un alumno. Este proceso de elaboración pragmática es esencial para la psicología y la didáctica, como es esencial para la historia de las ciencias" (Vergnaud, 1990, pág. 135).

Posteriormente, una vez que los estudiantes hayan resuelto sus tareas individuales, se propone una discusión colectiva sistemática desde la que se institucionalicen los conocimientos matemáticos. Por ello debemos fomentar un "aprendizaje intencionado" (Scardamalia et al., 1989), o aprendizaje en el que la construcción del conocimiento sea un proceso abierto y que los estudiantes tomen responsabilidades sobre el mismo; hay que favorecer un clima de trabajo en el que los estudiantes puedan examinar sus propios errores, que tengan oportunidades para el diálogo, que el clima de la clase esté libre de presiones externas y que los estudiantes acepten que la comprensión de los Números Racionales exige de un esfuerzo personal importante y de un tiempo amplio para la acomodación de los nuevos conocimientos con los que ya tenían (Hatano e Inagaki, citados por Sowder et al, 1995, pág. 258).

\section{A modo de balance}

Desde el curso 98/99 esta propuesta didáctica sobre el número racional está incorporada como parte de la asignatura "El currículo de Matemáticas en Educación Primaria", de 8 créditos. A lo largo de estos años se han detectado potencialidades y limitaciones de la propuesta que han aconsejado introducir las modificaciones que se señalan para mejorar la comprensión de los futuros Maestros sobre los números racionales positivos: 
1. Incrementar la presencia de materiales manipulativos en el aula.

Hemos detectado que los estudiantes ofrecen una gran resistencia a ampliar el significado de la fracción desde su conocimiento como relación parte-todo. En la resolución de tareas en las que hay que trabajar con otros significados, como medida o cociente, los alumnos tienden a trabajar con el significado parte-todo, lo que obstaculiza la correcta resolución de dichas tareas. Con la intención de ayudarles a superar este obstáculo hemos incrementado la presencia de objetos físicos en el aula de manera que, si el alumno lo necesita, pueda construir ideas sobre los otros significados de la fracción a partir de la observación de la realidad.

\section{Potenciar la construcción de conceptos.}

Una dilatada experiencia como aprendices ha situado a nuestros alumnos en la creencia de que el conocimiento de los números racionales se limita al uso de técnicas. En cada uno de los modelos de aprendizaje se proponen tareas sobre los diferentes significados tanto de los números racionales, como de las relaciones y operaciones entre ellos. Además, también se proponen tareas de justificar un mismo resultado matemático en los modelos medida y cociente, destacando las diferencias existentes en los argumentos utilizados en cada uno de dichos modelos.

3. Incrementar el trabajo con números periódicos.

El modelo medida obstaculiza la conceptualización de ideas sobre números periódicos pues la realización física del proceso de medida conlleva la idea de un número finito de pasos, no se concibe la medida como un proceso infinito. Si bien los alumnos admiten la existencia de números muy pequeños, tales situación se desvanece cuando aparece el número medida, pues el alumno se resiste a admitir el trabajo con cantidades de magnitud muy pequeñas. Nos encontramos, por tanto, con alumnos que no consiguen superar el obstáculo epistemológico que constituyen el infinito actual y el infinito potencial, puesto que trabajamos con esquemas de pensamiento construidos a partir de la experiencia práctica y adaptados a objetos y procesos finitos. Aun cuando este conflicto no es fácil de solucionar (Romero, 1995), hemos optado por incrementar el número de tareas, tanto de orden como de cálculo, en las que intervienen números periódicos, con la finalidad de que el alumno tenga mayor número de oportunidades para modificar sus esquemas mentales.

\section{BIBLIOGRAFÍA}

BALL, D.L. (1993) Halves, pieces, and twoths: constructing and using representational contexts in teaching fractions. En CARPENTER, T. P.; FENNEMA, E. y ROMBERG, T. A. (eds.): Rational Numbers. An integration of Research. Hilldale, N. J.: Lawrence Erlbaum Associates Publishers.

BOYER, C.B. (1986) Historia de la Matemática. Madrid: Alianza Editorial.

BROUSSEAU, G. (1993) Problemas en la enseñanza de los decimales. Problemas de didáctica de los decimales. Córdoba, Argentina: Universidad de Córdoba. 
BROWN, C.A. (1993) A critical analysis of teaching rational number. En CARPENTER, T. P.; FENNEMA, E. y ROMBERG, T. A. (eds.): Rational Numbers. An integration of Research. Hilldale, N. J.: Lawrence Erlbaum Associates Publishers.

CASTRO, E.; RICO, L. y ROMERO, I. (1997): Sistemas de representación y aprendizaje de estructuras numéricas. Enseñanza de las Ciencias, 15 (3), 361-371.

ESCOLANO, R. y GAIRÍN, J.M. (2004) Los modelos de medida en la enseñanza del número racional positivo en Educación Primaria: una propuesta alternativa a la relación parte-todo. En DEPARTAMENTO DE DIDÁCTICA DE LAS MATEMÁTICAS: Homenaje al profesor Jorge Cázares. Universidad de Granada (Pendiente de publicación).

GAGATSIS, A. y PATRONIS, T. (1990) Using Geometrical models in a process of reflective thinking in learning and teaching mathematics. Educational Studies in Mathematics, 21, 29-54.

GAIRÍN, J.M. (1999) Sistemas de representación de números racionales positivos. Un estudio con Maestros en formación. Tesis Doctoral, Universidad de Zaragoza.

GAIRÍN, J.M. (2001a) Modelos y significados. Conferencia pronunciada en los Cursos de Verano de El Escorial.

GAIRÍN, J.M. (2001b) Sistemas de representación de números racionales positivos: un estudio con maestros en formación. Contextos educativos, 4,137-159.

GIMÉNEZ, J. (1991) Innovación metodológica de la didáctica especial del número racional positivo. Diagnosis cognitiva y desarrollo metodológico. Tesis doctoral, Universidad Autónoma de Barcelona.

HIEBERT, J.A. (1993) Benefits and costs of research that links teaching and learning mathematics. En CARPENTER, T.P.; FENNEMA, E. y ROMBERG, T.A. (eds.): Rational Numbers. An integration of Research. Hilldale, N. J.: Lawrence Erlbaum Associates Publishers.

KERSLAKE, D. (1986) Fractions: Children's strategies and errors. Windsor, England: NFER-NELSON.

KIEREN, T.E. (1993) Rational and Fractional Numbers: From Quotient Fields to Recursive Understanding". En CARPENTER, T. P.; FENNEMA, E. y ROMBERG, T. A. (eds.): Rational Numbers. An integration of Research. Hilldale, N. J.: Lawrence Erlbaum Associates Publishers.

LESH, R. (1997): Matematización: La necesidad "real" de la fluidez en las representaciones. Enseñanza de las Ciencias, 15 (3), 377-391.

LESH, R.; POST, T. y BEHR, M. (1987) Representacions and translation among representation en Mathematics learning and Problem Solving. En JANVIER, C. (eds.): Problems of Representation in the teaching and learning of Mathematics. Hilldale, N. J.: LEA.

LLINARES, S. y SÁNCHEZ, M.V. (1996): Comprensión de las nociones matemáticas y modos de representación. El caso de los números racionales para profesores de Primaria. En GIMÉNEZ, J.; LLINARES, S. y SÁNCHEZ, M.V. (eds.): El proceso de llegar a ser profesor de primaria. Cuestiones desde la educación matemática. Granada: Colección Mathema. 
LLINARES, S.; SÁNCHEZ, M.V. y GARCÍA, M. (1994): Conocimiento de Contenido Pedagógico del Profesor. Tareas y modos de representación para las fracciones. Revista de Educación, 304, 199-225.

MARCK, N.K. (1995) Confounding Whole-Number and Fraction Concepts When Building on Informal Knowledge. Journal for Research in Mathematics Education, 26 (5), 422-441.

NATIONAL COUNCIL OF TECHERS OF N.C.T.M. (1989): Curriculum and evaluation standars for school mathematics. Reston, Virginia.

NATIONAL RESEARCH COUNCIL (1989) Everybody Counts: A report to the nation on the future of Mathematics education. Washington: National Academic Press.

NOVILLIS-LARSON, C. (1980) Locating proper fraction on number lines: Effect of length and equivalence. School Science and Mathematics, 53(5), 423-428.

OWENS, D.T. y SUPER, D.B., (1993): Teaching and Learning Decimal Fractions. En OWENS, D. (eds.): Research ideas for the classroom. Middle grades mathematics. Reston, Virginia: National Council of Teachers of Mathematics.

PHILIPPOU, G. y CHRISTOU, C. (1994): Prospective elementary Teachers'Conceptual and Procedural Knowledge of Fractions. En Proceedings XVIII PME. Lisboa, Portugal.

PINTO, M. y TALL, D. (1996): Student teachers'conceptions of the rational numbe". Proceedings XX PME. Valencia, España.

POST, T.R.; HAREL, G.; BEHR, M.J. y LESH, R. (1991): Intermediate teachers'knowledge of rational number concept. En FENNEMA, E.; CARPENTER, T.P. y LAMON, S.J. (eds.): Integrating research on teaching and learning mathematics. Albany, New York: SUNY.

RESNICK, L.B.; NESHER, P.; LEONARD, F.; MAGONE, M.; OMANSON, S. y PELED, I. (1989): Conceptual bases of artihmetic errors: the case of decimal fracctions. Journal for Research in Mathematics Education, 20, 8-27.

SÁNCHEZ VIDAL, B. (1866): Lecciones de aritmetica. Madrid, Imprenta de F. Martínez García.

SCARDAMALIA, M.; BEREITER, C.; MCLEAN, R.S.; SWALOW J. y WOODRUFF, E. (1989): Computer-supported intentional learning enviroments. Journal of Educational Computing Research, 5 (1), 51-68.

SIMON, M. (1993): "Prospective elementary Teachers' Kowledge of división". Journal for Research in Mathematics Education, 24 (3), 233-254.

SOWDER J.T. (1995) Instructing for rational number sense. En SOWDER, J.T. y SHAPPELLE, B.P. (eds.): Providing a Foundation for Teaching Mathematics in the Middle Grades. New York: State University of New York Press.

SOWDER, J.T.; BEZUK, N. y SOWDER, L.K. (1993): Using principles from cognitive psychology to guide rational number instruction for prospective teachers. En CARPENTER, T. P., FENNEMA, E. y ROMBERG, T. A. (eds.): Rational Numbers. An integration of Research. Hilldale, N. J.: Lawrence Erlbaum Associates Publishers.

SOWDER, J.T.; PHILIPP, R.A.; FLORES, A. y SCHAPPELLE, B.P. (1995) Instructional effects of kowledge of and about mathematics. a case study. En SOWDER, J.T. y SCHAPPLLE, B.P. (eds.): Providing a Foundation for Teaching Mathematics in the Middle Grades. New York: State University of New York Press. 
STREEFLAND, L. (1991) Fractions in realist mathematics education. A paradigm of developmental research. Dordrecht: Kluwer Academic Publishers.

THOMPSON, P. y THOMPSON, A. (1994) Talking About Rates Conceptually, Part I: A Teacher's Strunggle. Journal for Research in Mathematics Education, 25 (3), 279-303.

THOMPSON, P. y THOMPSON, A. (1996) Talking About Rates Conceptually, Part II: Mathematical Knowledge for Teaching. Journal for Research in Mathematics Education, 27 (1), 2-24.

VERGNAUD, G. (1990) Théorie des champs conceptuels. Recherches en Didactique des Mathématiques, 10, 47-56. 